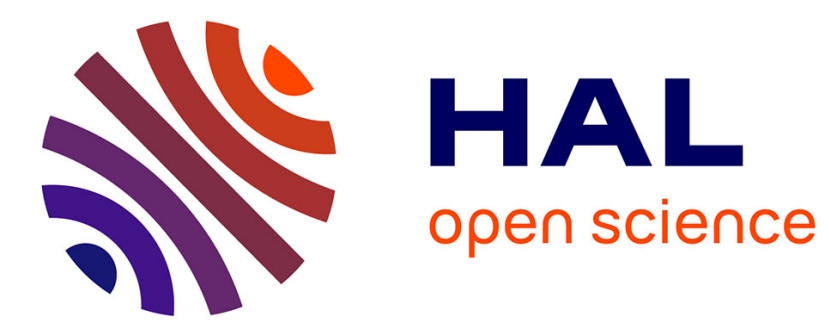

\title{
Critical current of granular superconductors
}

G. Deutscher

\section{- To cite this version:}

G. Deutscher. Critical current of granular superconductors. Revue de Physique Appliquée, 1973, 8

(2), pp.127-129. 10.1051/rphysap:0197300802012700 . jpa-00243667

\section{HAL Id: jpa-00243667 https://hal.science/jpa-00243667}

Submitted on 1 Jan 1973

HAL is a multi-disciplinary open access archive for the deposit and dissemination of scientific research documents, whether they are published or not. The documents may come from teaching and research institutions in France or abroad, or from public or private research centers.
L'archive ouverte pluridisciplinaire HAL, est destinée au dépôt et à la diffusion de documents scientifiques de niveau recherche, publiés ou non, émanant des établissements d'enseignement et de recherche français ou étrangers, des laboratoires publics ou privés. 
Classification

Phvsics Abstracts

14.24

\title{
CRITICAL CURRENT OF GRANULAR SUPERCONDUCTORS
}

\author{
G. DEUTSCHER (*) \\ Department of Physics and Astronomy \\ Tel-Aviv University, Tel-Aviv, Israël
}

(Reçu le 29 novembre 1972)

\begin{abstract}
Résumé. - Nous présentons le calcul du courant critique d'un système multicouches composé de films minces séparés par des barrières Josephson. On montre que pour des barrières très minces le courant critique est inversement proportionnel à la racine carrée de la résistivité effective, tandis que pour des barrières plus épaisses il est inversement proportionnel à la résistivité.
\end{abstract}

Abstract. - The transverse critical current of a multilayer system made of thin films separated by Josephson barriers is calculated. It is shown that for very thin barriers, the critical current is inversely proportional to the square root of the effective resistivity, whereas, for thicker barriers it is inversely proportional to the resistivity.

1. Introduction. - The structure of granular superconductors has been recently studied in some detail [1]. They consist of small cristallites, whose dimension (typically of the order of $50 \AA$ ) can be fairly uniform, and of a usually amorphous dielectric. Even for a rather low dielectric concentration, the normal state resistivity of granular materials $(>100 \mu \Omega . \mathrm{cm})$ is much higher than that of a typical alloy $(\sim 10 \mu \Omega . \mathrm{cm})$. It is therefore assumed that the small cristallites are surrounded by a thin dielectric coating which acts as a tunneling barrier between neighbouring cristallites.

This representation of a granular superconductor as a collection of weakly coupled small cristallites has already proved useful for the study of fluctuation effects. We shall use it here for the calculation of the critical current density, which is a matter of some practical interest since high normal state resistivity superconductors are required to build satisfactory power switches.

For this purpose, we can assume that the superconductor is made of metallic layers of thickness $L$ and surface $S$ separated by dielectric barriers whose resistance is called $R_{\mathrm{n}}$; the current is supposed to flow along the direction perpendicular to the layers. We shall neglect all fluctuation effects. i. e. we assume that the pair potential in each layer has a well defined amplitude $\Delta$ and phase, and therefore the dielectric

(*) On leave from Université de Paris Sud, Centre d’Orsay. layers parallel to the current flow don't play any role in this problem.

In short, we assume that the granular material can be represented by a series of Josephson junctions. It is well known that the critical current of one individual junction is inversely proportional to $R_{\mathrm{n}}$, and it would seem natural to assume that the same result applied to the granular material. However, we shall show here that, when $L$ is smaller than the coherence length, this result is in fact only obtained in the limit of large $R_{\mathrm{n}}$. In the limit of small $R_{\mathrm{n}}$ [2], [3], the critical current density is inversely proportional to $\left(R_{\mathrm{n}}\right)^{1 / 2}$. This calculation is presented in part 2 , whereas its practical implications are discussed in part 3.

2. Critical current of a series of Josephson junctions. - We assume that $L<\xi(T)$, where $\xi(T)$ is the temperature dependent coherence length in each individual metallic layer. The pair potential has therefore a constant amplitude $\Delta_{i}$ and phase $\varphi_{i}$ within each layer. If we further neglect all thermodynamic fluctuation of the $\Delta_{i}$ 's and $\varphi_{i}$ 's, all the $\Delta_{i}$ 's are equal and the phase difference $\varphi$ between adjacent layers is given by

$$
J=\frac{\pi}{4 e R_{\mathrm{n}}} \frac{\Delta^{2}}{k_{\mathrm{B}} T_{\mathrm{c}}} \sin \varphi
$$

where $J$ is the current, flowing perpendicular to the functions. 
The free energy density is given by

$$
\begin{aligned}
F_{\mathrm{s}}=F_{\mathrm{n}}+A \Delta^{2}+\frac{B}{2} \Delta^{4}+ & \frac{1}{L S} \frac{1}{R_{\mathrm{n}}} \frac{\pi \hbar}{8 e^{2}} \times \\
& \times \frac{\Delta^{2}}{k_{\mathrm{B}} T_{\mathrm{c}}}(1-\cos \varphi) .
\end{aligned}
$$

For a given value of $\varphi$, we now write that $\partial F_{\mathrm{s}} / \partial \Delta=0$ to obtain $\Delta(\varphi)$ :

$$
A+B \Delta^{2}+\frac{1}{L S} \frac{1}{R_{\mathrm{n}}} \frac{\pi \hbar}{8 e^{2}} \frac{1}{k_{\mathrm{B}} T_{\mathrm{c}}}(1-\cos \varphi)=0 .
$$

Using now (1) to express $\varphi$ as a function of $J$ and $\Delta$, we have

$$
\begin{aligned}
J^{2}=-J_{\mathrm{j}} \frac{4 e}{\hbar} L S A \Delta_{0}^{2} & f^{4}\left(1-f^{2}\right)\left(\frac{2 e}{\hbar}\right)^{2} \times \\
& \times(L S)^{2} \Delta_{0}^{4} A^{2} f^{4}\left(1-f^{2}\right)^{2}
\end{aligned}
$$

where $J_{\mathbf{j}}$ is the maximum Josephson current

$$
J_{\mathrm{j}}=\frac{\pi}{4 e R_{\mathrm{n}}} \frac{\Delta_{0}^{2}}{k_{\mathrm{B}} T_{\mathrm{c}}}
$$

$\Delta_{0}$ being the equilibrium value of $\Delta$ in zero current, and

$$
f=\frac{\Delta}{\Delta_{0}}
$$

Remembering that

$$
A=-\frac{1}{2} N(0)\left(\frac{\xi(0)}{\xi(T)}\right)^{2}
$$

where $\xi(T)$ is the temperature dependent coherence length [5], it is useful to introduce the quantity

$$
J(T)=\frac{2 e}{\hbar} L S \frac{1}{2} N(0) \Delta_{0}^{2}\left(\frac{\xi_{0}}{\xi(T)}\right)^{2}
$$

which is closely connected to the condensation energy $L S \frac{1}{2} N(0) \Delta_{0}^{2}$ per layer. Then (4) takes the simpler form

$$
J^{2}=J_{\mathrm{j}} J(T) f^{4}\left(1-f^{2}\right)\left[2-\frac{J(T)}{J_{\mathrm{j}}}\left(1-f^{2}\right)\right] .
$$

The critical current $J_{\mathrm{M}}$ is obtained by writing that $\mathrm{d} J^{2} / \mathrm{d} f^{2}=0$. Defining $c=J(T) / J_{\mathrm{j}}$ this condition reads :

$$
f_{\mathrm{M}}^{2}=\frac{3\left(c^{2}-c\right)+c \sqrt{c^{2}-2 c+9}}{4 c^{2}} .
$$

Since $f^{2}$ has to be positive, only the + sign applies in (9).

In the limit where $c \gg 1$ (very weakly coupled grains), $J_{M}$ may be written as

$$
J_{M}=J_{j}\left(1-\frac{1}{c}+\frac{1}{2} \frac{1}{c^{2}}+0\left(\frac{1}{c^{3}}\right)\right)
$$

which shows how $J_{M}$ goes to the Josephson value in the weak coupling limit, where the product $J_{\mathrm{M}} \cdot R_{\mathrm{n}}$ is a constant.

However, in the limit where $c \ll 1$ one obtains

$$
J_{M}^{2}=\frac{8}{27} J_{\mathrm{j}} J(T)\left(1-\frac{2}{9} c+0\left(c^{2}\right)\right) .
$$

Here, it is the product $J_{\mathrm{M}}^{2} \cdot R_{\mathrm{n}}$ which is constant. This result is similar to that obtained for a homogeneous superconductor containing non magnetic impurities. Figure 1 gives the shape of the general solution of (8), (9).

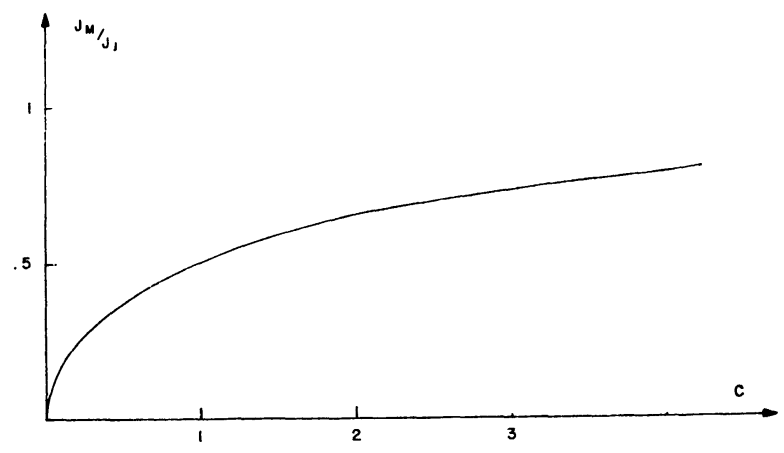

Fig. 1. - Critical current $J_{M}$ of a granular superconductor as a function of the intergrain coupling parameter $c$. In the weak coupling limit $(c \gg 1), J_{M}$ tends to the Josephson value $J_{\mathfrak{j}}$, whereas in the good coupling limit $(c \ll 1) J_{M}$ is equal to the critical current of an homogeneous alloy with the same resistivity.

3. Application to the case of granular superconductors. - Let us first evaluate the effective resistivity, $\rho_{\mathrm{c}}$, of a granular film where $\mathrm{c}=1$ :

$$
\frac{\pi}{4 e} \overline{R_{\mathrm{n}}} \frac{\Delta_{0}^{2}}{k_{\mathrm{B}} T_{\mathrm{c}}}=\frac{2 e}{\hbar} L S \frac{1}{2} N(0) \Delta_{0}^{2} \frac{\xi_{0}^{2}}{\xi(T)}
$$

where $L$ is now the grain size. Assuming that the grains are essentially pure, the effective resistivity of the granular material is $\rho_{\mathrm{n}}=R_{\mathrm{n}}(S / L)$ and we obtain at low temperatures

$$
\rho_{\mathrm{c}}=\frac{\pi \hbar}{4 e^{2}} \frac{L}{n} \frac{E_{\mathrm{F}}}{k_{\mathrm{B}} T_{\mathrm{c}}}
$$

where $n$ is the number of electrons per grain. For a grain size $L \sim 50 \AA, T_{\mathrm{c}} \sim 2 \mathrm{~K}$ and $E_{\mathrm{F}} \sim 3 \mathrm{eV}$, one obtains for $\rho_{\mathrm{c}}$ the typical value $10^{-2} \Omega . \mathrm{cm}$.

Hence, if $\rho_{\mathrm{n}} \ll 10^{-2} \Omega$.cm the granular film falls into the good coupling limit and eq. (11) applies. The critical current density $J_{\mathrm{c}}$ can then be written as

$$
J_{\mathrm{c}}^{2}=\frac{H_{\mathrm{c}}^{2}}{17.64} \bar{\rho}_{\mathrm{n}} \cdot \frac{k_{\mathrm{B}} T_{\mathrm{c}}}{\hbar} \cdot \frac{T_{\mathrm{c}}-T}{T_{\mathrm{c}}}
$$

where $H_{\mathrm{c}}$ is the bulk thermo-dynamical critical field at the temperature under consideration. Eq. (14) predicts that with high $T_{\mathrm{c}}$ superconductors, values of $\rho_{\mathrm{n}} J_{\mathrm{c}}^{2}$ up to $10^{11} \mathrm{~W} . \mathrm{cm}^{3}$ could in principle be obtained, with $\rho_{\mathrm{n}} \leqslant 10^{-2} \Omega . \mathrm{cm}$. This result might 
be quite interesting in practice [6]. The product $\rho_{\mathrm{n}} J_{\mathrm{M}}^{2}$ should be constant for a given temperature, and $J_{M}$ should vary as $\left(T_{\mathrm{c}}-T\right)^{3 / 2}$ near $T_{\mathrm{c}}$.

For $\rho_{\mathrm{n}}>10^{-2} \Omega$.cm, the situation is more complicated, since fluctuation effects cannot be neglected, as shown previously. Generally speaking, these fluctuation effects will reduce the critical current density and eq. (10) should be considered only as an upper bound for $T_{\mathrm{c}}$. More specifically, two cases have to be considered

1) If

$$
L^{3}>\frac{k_{\mathrm{B}} T_{\mathrm{c}}}{2 N(0)|\Delta(0)|^{2}},
$$

only the fluctuations in the phase difference between adjacent grains are important, but the order parameter has a well defined amplitude in each grain. The main square value of this phase difference is given by

$$
<\Phi^{2}>=\frac{k_{\mathrm{B}} T_{\mathrm{c}}}{\left|\Delta_{0}\right|^{2}} \frac{8 e^{2} \rho_{\mathrm{n}}}{\pi \hbar L} .
$$

At low enough temperatures, $\left\langle\Phi^{2}\right\rangle$ becomes small and the result (10) should be recovered.

2) If

$$
L^{3}<\frac{k_{\mathrm{B}} T_{\mathrm{c}}}{N(0)|\Delta(0)|^{2}}
$$

there are very large fluctuations in the amplitude of the order parameter and superconductivity essentially vanishes.

4. Conclusion. - In the good coupling limit, the critical current density of a granular superconductor is identical to that of an homogeneous alloy with the same resistivity, as already found for other properties by Parmenter [2] and Abeles et al. [3]. In the weak coupling limit, the Josephson result is recovered, provided that the grain size is large enough to prevent the destruction of the superconducting state by fluctuation effects. The crossover region occurs for normal state resistivities of the order of $10^{-2} \Omega . \mathrm{cm}$.

\section{References}

[1] Deutscher, G., Fenichel, H., Gershenson, M., Grunbaum, E. and Ovadyahu, Z., to be published in J. Low. Temp. Phys.

[2] Parmenter, R. H., Phys. Rev. 167 (1968) 387.

[3] Abeles, B., Cohen, R. W. and Cullen, G. W., Phys. Rev. Lett. 18 (1967) 902.
[4] JosePhson in « Superconductivity », (Parks R. D. ed., Marcel Dekker, N. Y.) 1969.

[5] DE GenNes, P. G. in " Superconductivity in Metals and Alloys », (p. 173) (Benjamin Inc., New York) 1966.

[6] Laquer, H. L., Ribe, F. L. and Veldon, D. M., Los Alamos Scientific Laboratory, Report 719145. 